\title{
Entering the Nickel Age of Superconductivity
}

\begin{abstract}
After a 30-year quest, researchers found a nickel-based analog of copper oxide superconductors. The discovery motivates the search for other nickelates and should provide new insights into the origin of high-temperature superconductivity.
\end{abstract}

\section{by Michael R. Norman ${ }^{1}$}

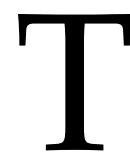
he 1986 discovery that a copper oxide compound could superconduct at a higher temperature than any material known at the time had a profound influence on physics. As reported by George Bednorz and Karl Muller, the material-made by chemically doping the oxide $\mathrm{La}_{2} \mathrm{CuO}_{4}$ - exhibited superconductivity at around $30 \mathrm{~K}$ [1]. Strikingly, whereas previously studied superconductors had tended to be nonmagnetic, the new material was derived from a magnetic insulator. Excitement about the new "high- $T_{c}$ " materials, which challenged existing theories of superconductivity, fueled the famous Woodstock of Physics at the American Physical Society's March Meeting of 1987 and graced the cover of Time Magazine two months later. Over the subsequent decades, transition temperatures of up to $165 \mathrm{~K}$ were observed in other copper oxides, or
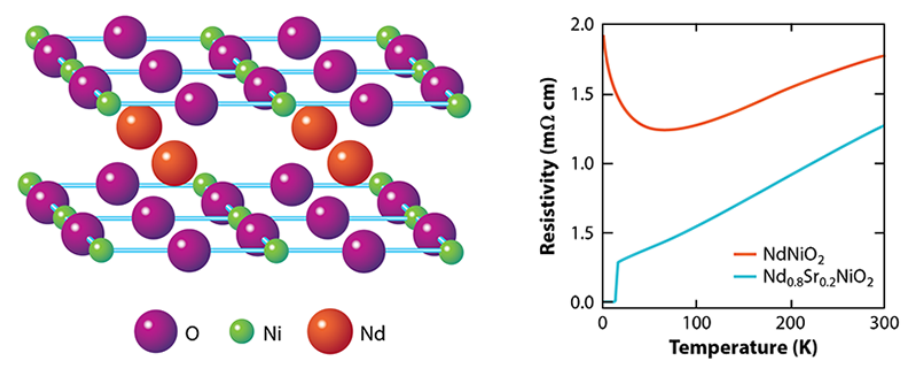

Figure 1: (Left) Crystal structure of the infinite-layer nickelate $\mathrm{NdNiO}_{2}$; the lines indicate the chemical bonds in the $\mathrm{NiO}_{2}$ planes. This material becomes superconducting when $\mathrm{Nd}$ is partially replaced by Sr. (Right) Resistivity of $\mathrm{NdNiO}_{2}$ (red) compared to $\mathrm{Nd}_{0.8} \mathrm{Sr}_{0.2} \mathrm{NiO}_{2}$ (blue) [2]. ((Left) APS/Carin Cain and M. Norman/Argonne National Lab. (Right) D. Li et al. [2]; adapted by APS/Carin Cain)

${ }^{1}$ Materials Science Division, Argonne National Laboratory, Lemont, IL, USA "cuprates."

Many structurally similar variants of these $3 d$-metal-ion oxides, some with copper, some without, have been discovered since 1986. Yet the only ones that superconduct contain copper oxide $\left(\mathrm{CuO}_{2}\right)$ planes, leaving physicists to wonder if there is something exclusive about cuprates. This outlook took a dramatic turn last August, when Harold Hwang and colleagues reported the first example of superconductivity in a material that contains planes of nickel oxide $\left(\mathrm{NiO}_{2}\right)$ instead [2]. Their observation would seem to offer new terrain for investigating models of high- $T_{\mathcal{C}}$ superconductors. But there are many issues to sort through in order to pinpoint the factors that unite and distinguish the nickel oxide and copper oxide superconductors. Such studies would also benefit greatly from the discovery of other nickel-containing superconductors, if researchers can overcome the many challenges to synthesizing them.

\section{A Nickel Oxide Superconductor, 20 Years in the Making}

The Hwang team's discovery did not come out of the blue. For decades, researchers have proposed other (non-copper) oxides as high- $T_{\mathcal{C}}$ candidates, the idea being that studying these cuprate analogs would lead to a better understanding of high-temperature superconductivity itself. In particular, the cuprates have several important ingredients, and there is much debate about which one is the most relevant for high $T_{c}$. First, in these materials, the copper ions give up two electrons $\left(\mathrm{Cu}^{2+}\right)$, leaving nine electrons to fill all but one of the ion's $3 d$ orbitals. In this $3 d^{9}$ configuration, the copper electrons couple strongly to the ligand-oxygen ions, leading to a strong "electron-lattice" interaction. Second, cuprates exhibit a small "charge-transfer energy" - the energy difference between the $3 d$ electronic states on copper and the $2 p$ states on oxygen. Third, the strong hybridization between copper and oxygen electrons and the small charge-transfer energy drive a large indirect interaction between the copper ions. This "superexchange" is a conjectured source for the anisotropic ( $d$-wave) nature of the cuprates' superconducting order parameter. To understand the relative importance 
of these three ingredients for superconductivity, the clear "knob" to turn is the choice of transition-metal ion that occupies copper's spot.

Nickel, which sits next to copper in the periodic table, is an obvious target. In 1999, Vladimir Anisimov and colleagues suggested that a strong candidate for a cuprate analog might be $\mathrm{LaNiO}_{2}$ [3], which they conjectured was a magnetic insulator and which had already been synthesized in the early 1980s but only superficially studied [4]. In this compound, nickel should be $\mathrm{Ni}^{1+}$, with a $3 d^{9}$ electronic configuration like $\mathrm{Cu}^{2+}$ in the cuprates. But Anisimov et al.'s assertion was challenged five years later by Kwan-Woo Lee and Warren Pickett, in a paper whose title provocatively claimed that " $\mathrm{Ni}^{1+}$ is not $\mathrm{Cu}^{2+"}$ [5]. Various groups looked at low-valence nickelates over the ensuing fifteen years, with Hwang's team finally achieving superconductivity in thin films of strontium-doped $\mathrm{NdNiO}_{2}$ with a $T_{c}$ of $\sim 10-15 \mathrm{~K}$ [2].

So, why the long wait? $\mathrm{NdNiO}_{2}$ and other materials of the form $R \mathrm{NiO}_{2}$ where $R$ is a rare-earth element, involve a series of tricky synthesis steps. The starting point is a different phase, $\mathrm{RNiO}_{3}$ with the so-called perovskite structure, in which the $\mathrm{Ni}$ ions are arranged in a quasicubic pattern as opposed to 2D layers. This precursor phase is already challenging to stabilize because it requires the $\mathrm{Ni}$ ions to be in an unfavorable, $3+$, valence state, whereas $\mathrm{Ni}$ prefers to be $2+$. The next step is to partially replace $R$, which is $3+$, by an ion that has a $2+$ valence, such as Sr. This substitution leads to $R_{1-x} \mathrm{Sr}_{x} \mathrm{NiO}_{3}$ where $x$ is the level of doping, and it pushes the $\mathrm{Ni}$ valence to an even more unstable value, $3+x$. The final step is to take out one-third of the oxygen ions using hydrogen as a reducing agent. This aggressive (and potentially damaging) chemical process turns the perovskite phase into the so-called infinite-layer $R_{1-x} \mathrm{Sr}_{x} \mathrm{NiO}_{2}$ phase, where $\mathrm{NiO}_{2}$ planes are separated by planes of $R / \mathrm{Sr}$ ions (Fig. 1, left). The Ni valence becomes $1+x$, giving rise to an electronic configuration similar to that of $\mathrm{Cu}$ in cuprates. But this valence is unfavorable for Ni when $x$ is small.

Hwang's group seem to have hit on the right synthesis recipe [2]. First, they stabilized the precursor perovskite by growing it in the form of a very thin $(\sim 100 \AA)$ film on a strontium titanate substrate. This thinness, along with the coupling to the substrate, helped stabilize the nickel oxide's structure during the hydrogen reduction step. They also discovered that in order to achieve superconductivity, lanthanum (the rare-earth element used in most earlier studies [4]) should be replaced by neodymium, which is smaller.

Nine months after their discovery, only one other group has reported similar results [6]. There are several potential reasons why. First, when attempting to grow the precursor perovskite phase, there is a danger of instead nucleating another phase ( $\left.R_{4-x} \mathrm{Sr}_{x} \mathrm{Ni}_{3} \mathrm{O}_{10}\right)$ with a more stable Ni valence [7]. Second, there is a fine line between fully reducing the film to the desired composition and over-reducing it, which can lead to sample decomposition. The reduction step also risks incorporating hydrogen into the sample, which acts as a dopant. These challenges become amplified when trying to grow bulk samples instead of films, and to date, no superconducting bulk nickel oxides have been reported.

\section{Are Nickelates Similar to Cuprates, or Something New?}

What are the theoretical implications of finding a superconducting nickelate [2]? Let's start with what is known about the magnetic and transport properties. Despite the assertion by Anisimov et al. [3], the undoped $\mathrm{RNiO}_{2}$ compounds did not turn out to be magnetic insulators as the undoped cuprates are. Instead, $\mathrm{RNiO}_{2}$ materials exhibit metallic-like behavior - with a resistivity that decreases with temperature-until about $100 \mathrm{~K}$. At this point, the resistivity starts to rise logarithmically with decreasing temperature (Fig. 1, right). This "upturn" is reminiscent of the Kondo effect seen in metals containing magnetic impurities, where electron scattering becomes enhanced at low temperatures. Strikingly, there's no evidence for magnetism in $\mathrm{RNiO}_{2}[8,9]$ despite the fact that calculations routinely predict its existence.

The previous work of Lee and Pickett offers a compelling explanation for the differences between undoped cuprates and nickelates [5]. In insulating cuprates, such as $\mathrm{R}_{2} \mathrm{CuO}_{4}$, the $5 d$ states associated with the rare-earth ions are empty and play no special role. But Lee and Pickett showed that in $\mathrm{RNiO}_{2}$, the $5 d$ states become partially occupied. The presence of the $5 d$ electrons is consistent with experiments, which reveal not only metallic behavior but also evidence for negative charge carriers (specifically, a negative Hall number) $[2,10,11]$. In principle, $5 d$ conduction electrons could screen the Ni spins, suppressing magnetism and giving rise to a Kondo effect like that seen in heavy fermion materials, as suggested in Ref. [12]. But whether the $5 d$ electrons provide significant screening remains controversial, and it's possible that the resistance upturn is simply due to weak localization.

Besides the presence of $5 d$ electrons, nickelates have some relevant differences from the cuprates that might elucidate the role of the high- $T_{c}$ "ingredients" noted above (Fig. 2). In both nickelates and cuprates, the $3 d$ orbitals of the metal ion hybridize with the $2 p$ orbitals on the oxygen sites. However, the $3 d$ states of nickel lie higher in energy than those of copper and, therefore, closer to the $5 d$ states of the rare-earth ion. This proximity is why the $5 d$ states are partially occupied. It also increases the charge-transfer energy between the $3 d$ and $2 p$ states relative to the cuprates. Because of this increase, the active band of electrons for $\mathrm{Ni}$ (the $x^{2}-y^{2}$ band) is narrower than that for $\mathrm{Cu}$, meaning that various electronic interactions should be enhanced. On the other hand, the increase in charge-transfer energy also suppresses the superexchange interaction, reducing the tendency for magnetism, but not 


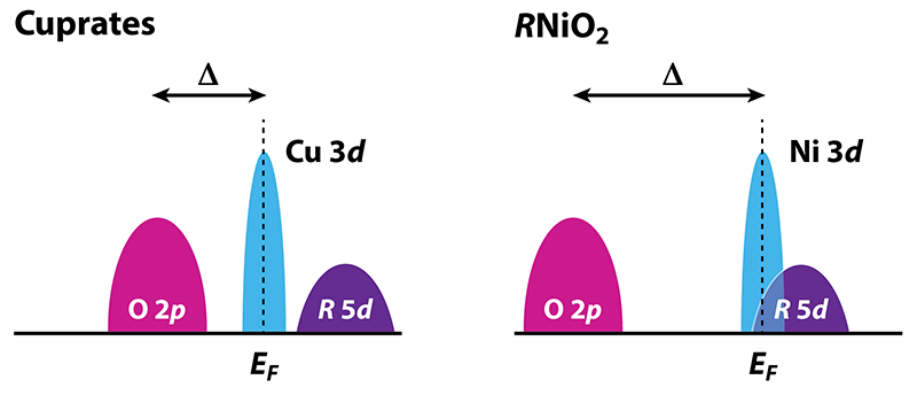

Figure 2: Schematic energy diagram of the relevant electrons in cuprates (left) and $\mathrm{RNiO}_{2}$ (right). $E_{F}$ is the Fermi energy, and $\Delta$ the charge-transfer energy between the $\mathrm{Cu}$ (or $\mathrm{Ni}$ ) $3 d$ states and the $\mathrm{O} 2 p$ states. Here, $3 d$ refers to the active $\left(x^{2}-y^{2}\right)$ orbital. Compared with cuprates, the $3 d$ states in $\mathrm{RNiO}_{2}$ move to a higher energy relative to the $\mathrm{O} 2 p$ states. This shift leads to a larger charge-transfer energy and partial occupancy of the $R 5 d$ states. (APS/Carin Cain and M. Norman/Argonne National Lab)

(according to calculations) eliminating it altogether. This suppressed superexchange may also explain why the $T_{\mathcal{C}}$ observed in $R_{1-x} \mathrm{Sr}_{x} \mathrm{NiO}_{2}$ is fairly low $(<15 \mathrm{~K})$ : as noted above, many theories for high- $T_{\mathcal{C}}$ in cuprates are based on either a small charge-transfer energy and/or a large superexchange. Further studies contrasting nickelates and cuprates should provide new insights into the origin and nature of hightemperature superconductivity.

\section{Are Other Superconducting Nickelates Out There?}

One way to explore the issues associated with the $5 d$ electrons is to make $R \mathrm{NiO}_{2}$ with $3+$ ions other than $\mathrm{Nd}[13,14]$, as this substitution would tune the relative energies of the $5 d$ and $3 d$ states. The Hwang group has already reported superconductivity for $R=\operatorname{Pr}$ (praseodymium) [15]. But much more could be learned from the discovery of other classes of superconducting nickelates. In this context, an interesting pathway to explore would be reduced versions of the so-called Ruddlesden-Popper (R-P) phases. With a chemical formula of $R_{n+1} \mathrm{Ni}_{n} \mathrm{O}_{2 n+2}$, these compounds contain $n$ $\mathrm{NiO}_{2}$ planes per formula unit. In particular, a promising option is $\mathrm{Pr}_{4} \mathrm{Ni}_{3} \mathrm{O}_{8}$, the $n=3$ reduced R-P phase (Fig. 3, left). Theoretical and experimental work in 2017 showed that $\mathrm{Pr}_{4} \mathrm{Ni}_{3} \mathrm{O}_{8}$ has a similar electronic structure and transport properties as an overdoped cuprate-that is, a cuprate that's been hole-doped beyond the level where superconductivity occurs [16]. Reaching the desired doping range for superconductivity in $\mathrm{Pr}_{4} \mathrm{Ni}_{3} \mathrm{O}_{8}$ (Fig. 3, right) might be possible through various means of electron doping, including replacing $R$ with a $4+$ ion (such as Ce), intercalating an electron-donating ion (such as $\mathrm{Li}$ ), or gating the material with an ionic liquid.

Superconductivity in the $\mathrm{Pr}_{4} \mathrm{Ni}_{3} \mathrm{O}_{8}$ family would be inter-
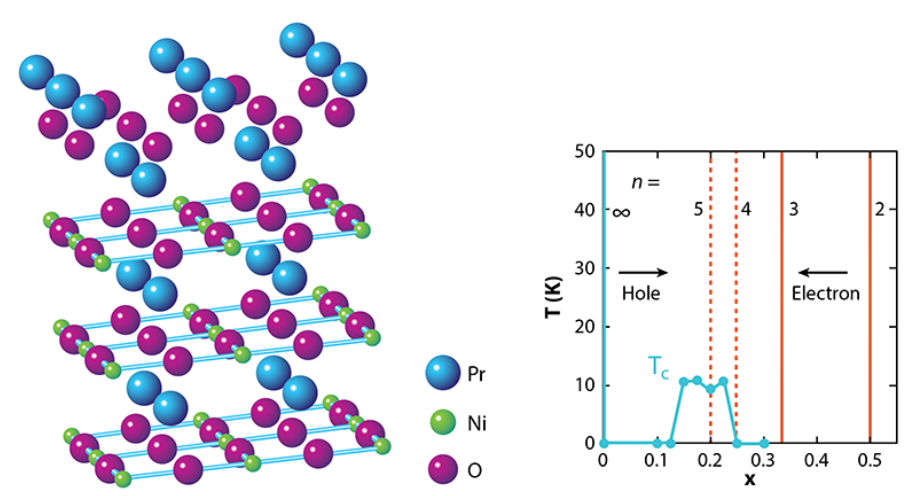

Figure 3: (Left) The reduced $n=3$ Ruddlesden-Popper phase $\mathrm{Pr}_{4} \mathrm{Ni}_{3} \mathrm{O}_{8}$, with three $\mathrm{NiO}_{2}$ planes per formula unit. (Right) Phase diagram of layered nickelates versus doping level $x$. The vertical lines show reduced R-P phases with different $n$, where $n$ is the number of $\mathrm{NiO}_{2}$ planes per formula unit and $x=1 / n$. The infinite-layer $\mathrm{RNiO}_{2}$ corresponds to $n=\infty$. Researchers have shown that hole-doping $\mathrm{NdNiO}_{2}$ by replacing $\mathrm{Nd}$ with $\mathrm{Sr}$ leads to superconductivity (blue dots) [2],[6],[7],[11]. According to predictions, electron-doping certain reduced R-P phases might also lead to superconductivity. (Dotted lines indicate reduced R-P phases that have yet to be reported.) (APS/Carin Cain and M. Norman/Argonne National Lab)

esting because these materials have completely depopulated rare-earth $5 d$ states. More importantly, their charge-transfer energies are intermediate between those of $\mathrm{RNiO}_{2}$ and cuprates. As a consequence, their superexchange interactions are likely larger, potentially leading to a higher $T_{c}$. As an example, $\mathrm{La}_{4} \mathrm{Ni}_{3} \mathrm{O}_{8}$ exhibits a phenomenon seen in some cuprates (stripe order) [17], and a fit of the magnon dispersion measured with $x$ rays was able to determine the superexchange energy [18]. The value of this energy appears to be intermediate between cuprates and that recently estimated for $\mathrm{NdNiO}_{2}$ [19].

Reduced versions of "higher-order" $(n>3)$ R-P phases would also be worth exploring. In these compounds, the doping level $x$ is equal to $1 / n$. Thus a reduced $n=5 \mathrm{R}-\mathrm{P}$ phase would have the same doping level, $x=0.2$, as the superconducting compound reported by Hwang's group [2] (Fig. 3, right). But it would not suffer from the disorder that's associated with strontium dopants.

\section{Directions for the Future}

Studying materials in thin-film form has limitations. Many of the methods that were useful for characterizing cuprates, such as neutron scattering, require bulk, preferably singlecrystal, samples. The availability of superconducting nickelate single crystals would also assuage the concern that the observed superconductivity might arise from an interface effect with the substrate [20, 21]. Bulk crystals would also be desirable for studying the material's phonons, whose role in 
high- $T_{\mathcal{C}}$ superconductivity has long been debated.

Entering the "nickel age" will be difficult. A high school student can make crude cuprate samples in a microwave oven. But preparing nickelates requires an involved process that only a few groups have the expertise to do. Still, with sufficient investment in materials synthesis, there is every reason to expect a wealth of samples in the future. Even if high-quality single crystals are slow to come for the $R \mathrm{NiO}_{2}$ compounds, we still have a lot to learn from these materials in thin-film form-for example, researchers successfully performed transport measurements, resonant x-ray scattering, and other important studies on thin films of the $\mathrm{RNiO}_{3}$ perovskites. Certainly, despite the limited experimental results to date, over thirty theory papers have appeared on these new $R \mathrm{NiO}_{2}$ materials since the Hwang group's announcement [2]. As discussed above, the ideas expressed in these papers range all the way from nickelates being chargetransfer materials like cuprates, with $\mathrm{Ni} 3 d$ and $\mathrm{O} 2 p$ as the active orbitals, to models where the relevant degrees of freedom are instead $\mathrm{Ni} 3 d$ and $R 5 d$, as in Kondo models of heavy fermion materials. Resolving this matter will not only help explain superconductivity in these new nickelates but will also give us better insight into the nature of hightemperature superconductivity in the cuprates.

\section{REFERENCES}

[1] J. G. Bednorz and K. A. Muller, "Possible high- $T_{\mathcal{C}}$ superconductivity in the Ba-La-Cu-O system," Z. Phys. B 64, 189 (1986).

[2] D. Li et al., "Superconductivity in an infinite-layer nickelate," Nature 572, 624 (2019).

[3] V. I. Anisimov et al., "Electronic structure of possible nickelate analogs to the cuprates," Phys. Rev. B 59, 7901 (1999).

[4] M. Crespin et al., "Reduced forms of $\mathrm{LaNiO}_{3}$ perovskite. Part 1.-Evidence for new phases: $\mathrm{La}_{2} \mathrm{Ni}_{2} \mathrm{O}_{5}$ and $\mathrm{LaNiO}_{2}$," J. Chem. Soc., Faraday Trans. 2 79, 1181 (1983).

[5] K.-W. Lee, and W. E. Pickett, "Infinite-layer $\mathrm{LaNiO}_{2}: \mathrm{Ni}^{1+}$ is not $\mathrm{Cu}^{2+}$," Phys. Rev. B 70, 165109 (2004).

[6] S. Zeng et al., "Phase diagram and superconducting dome of infinite-layer $\mathrm{Nd}_{1-x} \mathrm{Sr}_{x} \mathrm{NiO}_{2}$ thin films," arXiv:2004.11281.
[7] K. Lee et al., "Aspects of the synthesis of thin film superconducting infinite-layer nickelates," APL Mater. 8, 041107 (2020).

[8] M. A. Hayward et al., "Sodium hydride as a powerful reducing agent for topotactic oxide deintercalation: Synthesis and characterization of the Nickel(I) Oxide $\mathrm{LaNiO}_{2}$," Journal of the American Chemical Society 121, 8843 (1999).

[9] M. A. Hayward and M. J. Rosseinsky, "Synthesis of the infinite layer $\mathrm{Ni}(\mathrm{I})$ phase $\mathrm{NdNiO}_{2+x}$ by low temperature reduction of $\mathrm{NdNiO}_{3}$ with sodium hydride," Solid State Sci. 5, 839 (2003).

[10] A. S. Botana and M. R. Norman, "Similarities and differences between $\mathrm{LaNiO}_{2}$ and $\mathrm{CaCuO}_{2}$ and implications for superconductivity," Phys. Rev. X 10, 011024 (2020).

[11] D. Li et al., "Superconducting dome in $\mathrm{Nd}_{1-x} \mathrm{Sr}_{x} \mathrm{NiO}_{2}$ infinite layer films," arXiv:2003.08506.

[12] G.-M. Zhang et al., "Self-doped Mott insulator for parent compounds of nickelate superconductors," Phys. Rev. B 101, 020501 (2020).

[13] E. Been, "Theory of rare-earth infinite layer nickelates," arXiv:2002.12300.

[14] A. S. Botana and M. R. Norman, "Layered palladates and their relation to nickelates and cuprates," Phys. Rev. Materials 2, 104803 (2018).

[15] M. Osada et al., "A superconducting praseodymium nickel oxide with infinite-layer structure," Bull. Am. Phys. Soc., APS March Meeting 2020, Abstract J48.00004 http://meetings.aps. org/Meeting/MAR20/Session/J48.4.

[16] J. Zhang et al., "Large orbital polarization in a metallic squareplanar nickelate," Nat. Phys. 13, 864 (2017).

[17] J. Zhang et al., "Spin stripe order in a square planar trilayer nickelate," Phys. Rev. Lett. 122, 247201 (2019).

[18] The magnon dispersion was measured with resonant inelastic $\mathrm{x}$-ray scattering (Mark Dean, private communication).

[19] Y. Fu et al., "Core-level X-ray photoemission and Raman spectroscopy studies on electronic structures in Mott-Hubbard type nickelate oxide $\mathrm{NdNiO}_{2}$," arXiv:1911.03177.

[20] F. Bernardini and A. Cano, "Stability and electronic properties of the $\mathrm{LaNiO}_{2} / \mathrm{SrTiO}_{3}$ interface," arXiv:2001.02133.

[21] B. Geiser and R. Pentcheva, "Fundamental difference in the electronic reconstruction of infinite-layer vs. perovskite neodymium nickelate films on $\mathrm{SrTiO}_{3}(001)$," arXiv:2001.03762.

10.1103/Physics. 13.85 